\title{
Acute and chronic hemorrhage from radiation-induced cavernous malformation associated with late-delayed radiation necrosis in long surviving glioma patients: A case report
}

\author{
TOMOYA OISHI $^{1}$, TOMOHIRO YAMASAKI ${ }^{1}$, SATOSHI BABA $^{2}$, SHINICHIRO KOIZUMI $^{1}$, \\ TETSURO SAMESHIMA ${ }^{1}$ and HIROKI NAMBA ${ }^{1}$ \\ Departments of ${ }^{1}$ Neurosurgery and ${ }^{2}$ Diagnostic Pathology, \\ Hamamatsu University School of Medicine, Hamamatsu, Shizuoka 431-3192, Japan
}

Received April 3, 2019; Accepted July 1, 2019

DOI: $10.3892 / \mathrm{ol} .2019 .11113$

\begin{abstract}
Although radiation therapy is a standard treatment strategy for patients with glioma, its delayed complications are not clearly understood. Radiation-induced cavernous malformation (RICM) is one of the complications in the delayed phase following radiation therapy, which usually occurs in children. Herein we present three cases of RICM with radiation necrosis in long surviving adult glioma patients, 2 with oligoastrocytoma and one with anaplastic ependymoma. Two of three patients had received an obvious overdose of radiation by additional stereotactic radiation therapy. Repeated episodes of either acute or chronic hemorrhages from RICM worsened the neurological symptoms in all cases. The interval between the last irradiation and the occurrence of symptoms was 45-173 months. The presence of hypointense rim on FLAIR or $\mathrm{T} 2 *$ on magnetic resonance imaging, which resembles the appearance of sporadic cavernous malformations, could be helpful in differentiating RICM from tumor recurrence. Surgical resection was effective in alleviating the symptoms. Microscopically, RICM is a vascular lesion with vulnerable vessels, which are observed in the center of the radiation necrosis. Repeated hemorrhages from these vessels cause either gradual or sudden worsening of neurological symptoms. Therefore, radiation overdose, which results in radiation injury, should be avoided in low grade glioma patients, who could potentially survive for a long period.
\end{abstract}

Correspondence to: Dr Hiroki Namba, Department of Neurosurgery, Hamamatsu University School of Medicine, 1-20-1 Handayama, Hamamatsu, Shizuoka 431-3192, Japan

E-mail: hnamba@hama-med.ac.jp

Abbreviations: RICM, radiation-induced cavernous malformation; FLAIR, fluid attenuated inversion recovery; MRI, magnetic resonance imaging; CT, computed tomography; VEGF, vascular endothelial growth factor; HIF-1 $\alpha$, hypoxia inducible factor- $1 \alpha$

Key words: radiation necrosis, radiation-induced cavernous malformation, glioma, hemorrhage

\section{Introduction}

Radiation therapy is a less invasive treatment for brain and neck diseases, such as head and neck cancers and arteriovenous malformations (1-4). Although some studies reported the efficacy of stereotactic radiation therapy for either newly diagnosed glioma or recurrent glioma, especially for lesions that are unamenable to resection, such as lesions of eloquent areas of the brain, strong recommendation of stereotactic radiation therapy for glioma has not yet been established (5-7). Necrosis, cavernous malformation, and radiation-induced tumor formation are major side effects observed in the latter stages of radiation therapy; these side effects sometimes distress the patients (8-10). Mostly, radiation necrosis occurs about 3-36 months after radiation therapy (11-13), and radiation-induced cavernous malformation (RICM) caused by radiation injury is reported to occur after a rather longer period of 1-26 years after radiation therapy (9-11). Due to the long latency period of RICM after radiation therapy, RICMs occurring in glioma patients have been scarcely reported $(9,10,14)$.

Here, we present three cases of long surviving glioma patients with symptomatic hemorrhage from RICM, resulting from radiation necrosis that occurred more than 4 years after the last irradiation episode. The lesions were pathologically proven in 2 cases and clinically suggested by magnetic resonance imaging (MRI) in 1 case. Thus, it is important to consider late complications of radiation therapy when administered to low grade glioma patients, since they could potentially survive for a long period.

\section{Case report}

Case 1. At age 50 years, the female patient had a left insular tumor, which invaded the frontal and temporal lobes (Fig. 1A). The histopathological diagnosis of the biopsy specimen was oligoastrocytoma. Using a CyberKnife, the patient was initially administered $35 \mathrm{~Gy} / 5 \mathrm{fr}$ (Fig. 1B). At age 55 years, each lesion appeared in the temporal and insular cortex on contrast-enhanced MRI; the patient was re-treated with additional $30 \mathrm{~Gy} / 3 \mathrm{fr}$ (Fig. 1C and D), followed by temozolomide and interferon $\beta$ chemotherapy for 4 years. 

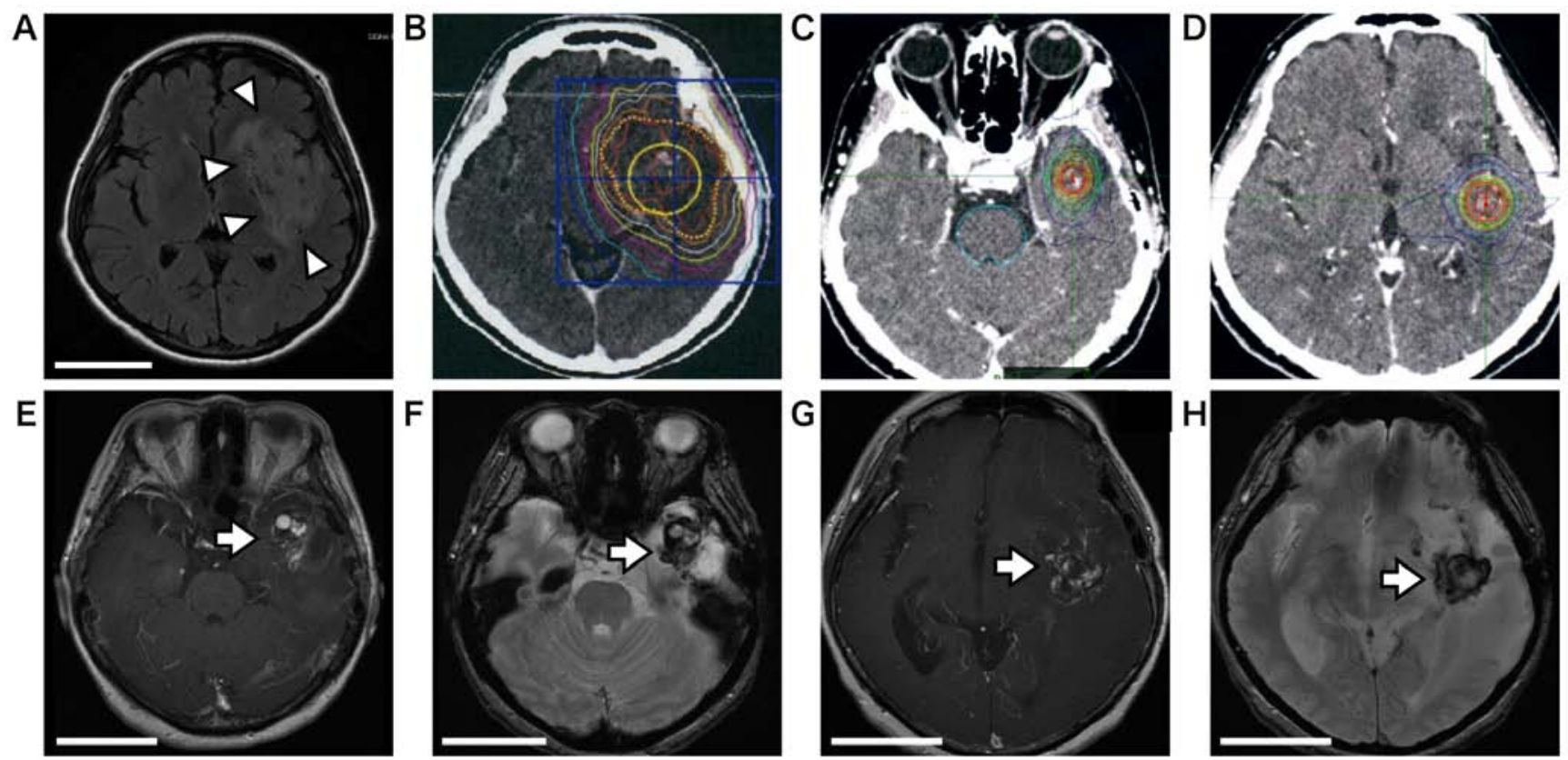

Figure 1. Case 1 (lesion 1 and 2). (A) The FLAIR image at the first presentation showed left insular lesion expanding into frontal and temporal lobe (arrow head). (B-D) Dose distribution of the first radiotherapy at the age of 50 (B) and the second radiotherapy at the age of 55 (C and D). (E and F) The contrastenhanced T1 weighted image (T1/CE) of the lesion 1 in the left temporal lobe at the age of 59 showed soap bubble sign (E) and the T2* image showed a hypo-intensity rim indicating peri-lesional hemorrhage (F) (arrow). ( $\mathrm{G}$ and $\mathrm{H}$ ) Two years later, a similar lesion appeared in the insula (lesion 2) (T1/CE; G and $\left.\mathrm{T} 2^{*}, \mathrm{H}\right)$ (arrow). Scale bar, $5 \mathrm{~cm}$.

At age 59 years, she presented with gradual development of speech disturbance and was referred to our hospital. An MRI scan revealed an enlargement of the temporal mass with heterogeneous enhancement on contrast-enhanced T1 weighted image (T1-CE) (Fig. 1E) and low intensity rim on $\mathrm{T}^{*}$ with strong perifocal edema (Fig. 1F). The lesion (lesion 1) was totally resected; histopathological examination of the lesion revealed cavernous malformation and radiation necrosis without tumor recurrence. The patient was closely observed without further chemotherapy.

Two years later, another MRI scan revealed a gradually increasing enhanced insular mass with minor hemorrhage and perifocal edema (Fig. 1G and H). RICM with radiation necrosis was suspected because of similarities in MRI findings to the previous ones. Since aphasia and right hemiparesis gradually worsened even after corticosteroid treatment, the lesion (lesion 2) was partially removed and the histopathological diagnosis was again radiation necrosis without tumor recurrence. A ventriculoperitoneal shunt was placed for concurrent communicating hydrocephalus. Perifocal edema gradually decreased; she could now perform daily activities at home without much support.

Case 2. At age 52 years, this male patient had a right occipital tumor (Fig. 2A). After total resection of the tumor, a histopathological examination revealed an anaplastic ependymoma. The patient was treated with $60 \mathrm{~Gy}$ of postoperative external irradiation (Fig. 2B). One year later, a small enhanced nodule on the tumor resection surface was noticed on MRI (Fig. 2C) and 20 Gy of $\gamma$-knife radiation was administered (Fig. 2D). Without additional treatment in 12 years, no enhanced lesion was observed.

At age 67 years, the patient presented with intermittent visual hallucinations in the left visual field. An MRI scan revealed a heterogeneous enhanced mass lesion (Fig. 2E) that enlarged gradually. The lesion was comprised of hemorrhages that consisted of low signals on T2* on MRI images (Fig. 2F). After total resection of the lesion (lesion 3), a histopathological examination revealed radiation necrosis with cavernous malformation-like areas. The postoperative course was uneventful and visual hallucination completely disappeared.

Case 3. At age 39 years, this male patient was diagnosed of left insular anaplastic astrocytoma with an oligodendroglial component by open biopsy in the previous hospital. The patient was treated with intraoperative irradiation (dose unknown) and $56 \mathrm{~Gy}$ of conventional external irradiation, followed by administration of four doses of nimustine hydrochloride. After finishing the initial treatment, the patient was referred to our hospital. An MRI scan revealed a T2 high lesion with no obvious enhancement after more than 10 years (Fig. 3A).

At age 50 years, another MRI scan revealed a cystic lesion (lesion 4) with mild enhancement in the insula (Fig. 3B and C). A biopsy was performed in suspicion of tumor recurrence, but a histopathological examination revealed only gliosis with no tumor recurrence. The patient did not receive further treatment but was assessed with MRI during follow-up. Two years later, the patient suffered a sudden onset of severe right hemiparesis and motor aphasia. Computed tomography (CT) scans revealed subcortical hemorrhage right on the primary motor cortex, adjacent to the tumor location (Fig. 3D). Two years later, the right hemiparesis suddenly worsened again; a CT scan revealed a hemorrhage recurrence in almost the same area of the previous hemorrhage. Although hematoma was stereotactically aspirated, the patient experienced severe right hemiparesis and mild aphasia. 

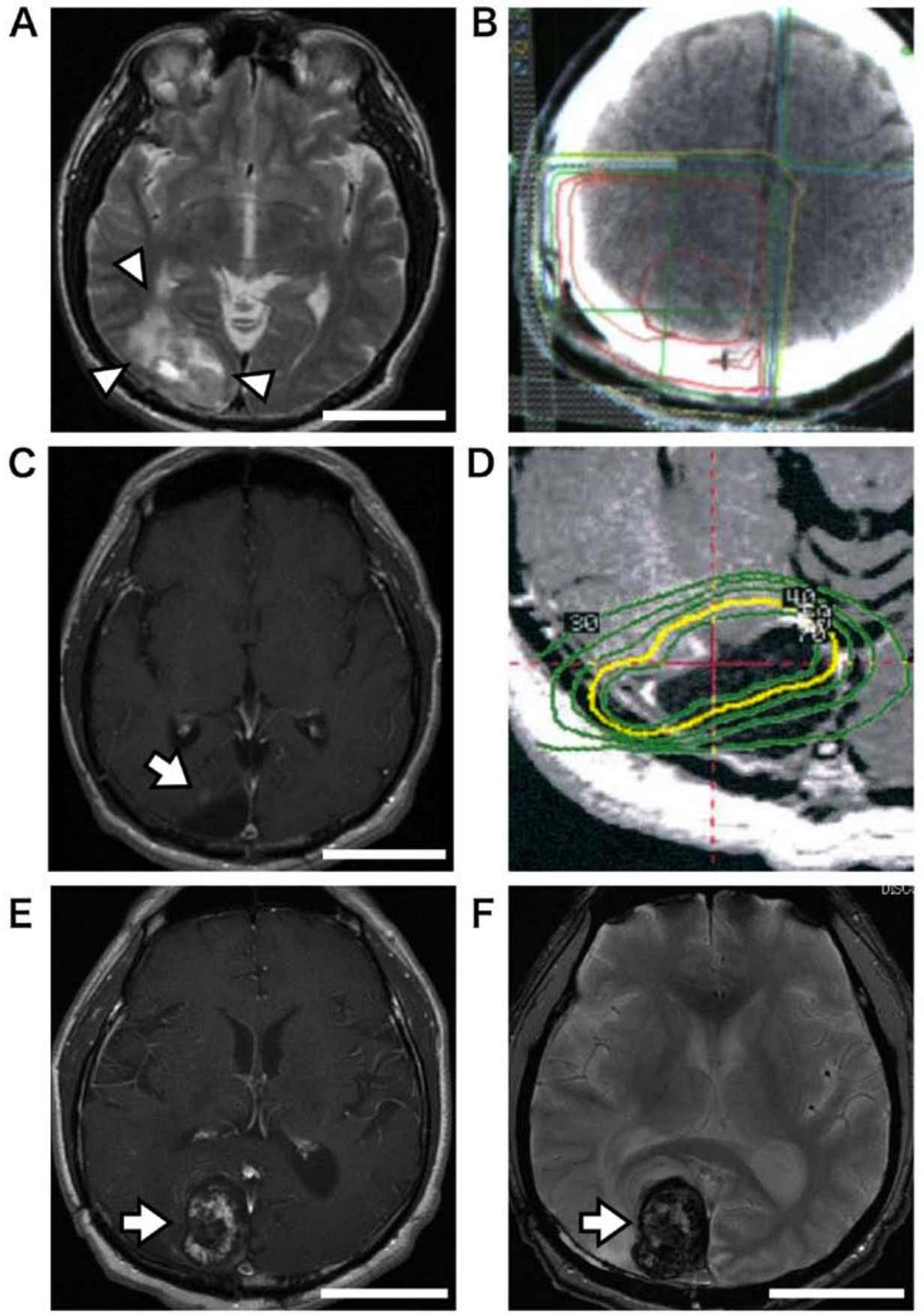

Figure 2. Case 2 (lesion 3). (A) The T2 weighted image at the first presentation showed right occipital mass with perifocal edema (arrow head). (B) Dose distribution of the adjuvant conventional radiotherapy. (C) Small enhanced nodule on T1/CE (arrow) appeared one year later. (D) The lesion was then treated with $\gamma$-knife. (E and F) The T1/CE image of the lesion 3 at the second presentation showing soap bubble sign (E) and the T2* image showing a hypo-intensity rim on $\mathrm{T} 2 *(\mathrm{~F})$ (arrow). Scale bar, $5 \mathrm{~cm}$.

Histological findings. Histopathological examination was performed on each surgically resected or biopsied specimen fixed with $10 \%$ formalin for $24 \mathrm{~h}$ at room temperature. Paraffin-embedded sections cut by $4 \mu \mathrm{m}$ thickness were performed hematoxylin and eosin (H\&E) and following immunohistological staining. For immunohistological examination, after deparaffinization, antigen activation was performed at $95^{\circ} \mathrm{C}$ for $40 \mathrm{~min}$ at $\mathrm{pH} 9.0$ using TE buffer. The commercially available primary antibodies, CD31 (monoclonal mouse; Dako, Agilent Technologies Inc.; clone: JC70A, code no. M0823; dilution, 1:30) and VEGF (vascular endothelial growth factor) (polyclonal rabbit; GeneTex Inc.; GTX102643 dilution, 1:100), were used at room temperature for $30 \mathrm{~min}$. Subsequently, ChemMate Envision kit/HRP (Dako, Agilent Technologies Inc.) for CD31 immunostaining and Histofine Simple Stain MAX-PO (Multi) (Nichirei Bioscience Inc.) for VEGF immunostaining were used as the secondary antibody at room temperature for $30 \mathrm{~min}$. Finally, sections were reacted with 3,3-Diaminobenzidine (DAB) as a chromogen at room temperature. Slides were observed under a light microscope.

Radiation necrosis was histologically diagnosed in all cases, except in case 3 which was diagnosed as gliosis. Microscopically, coagulative necrosis, hyalinized blood vessels, and telangiectasia, which is suggestive of radiation 

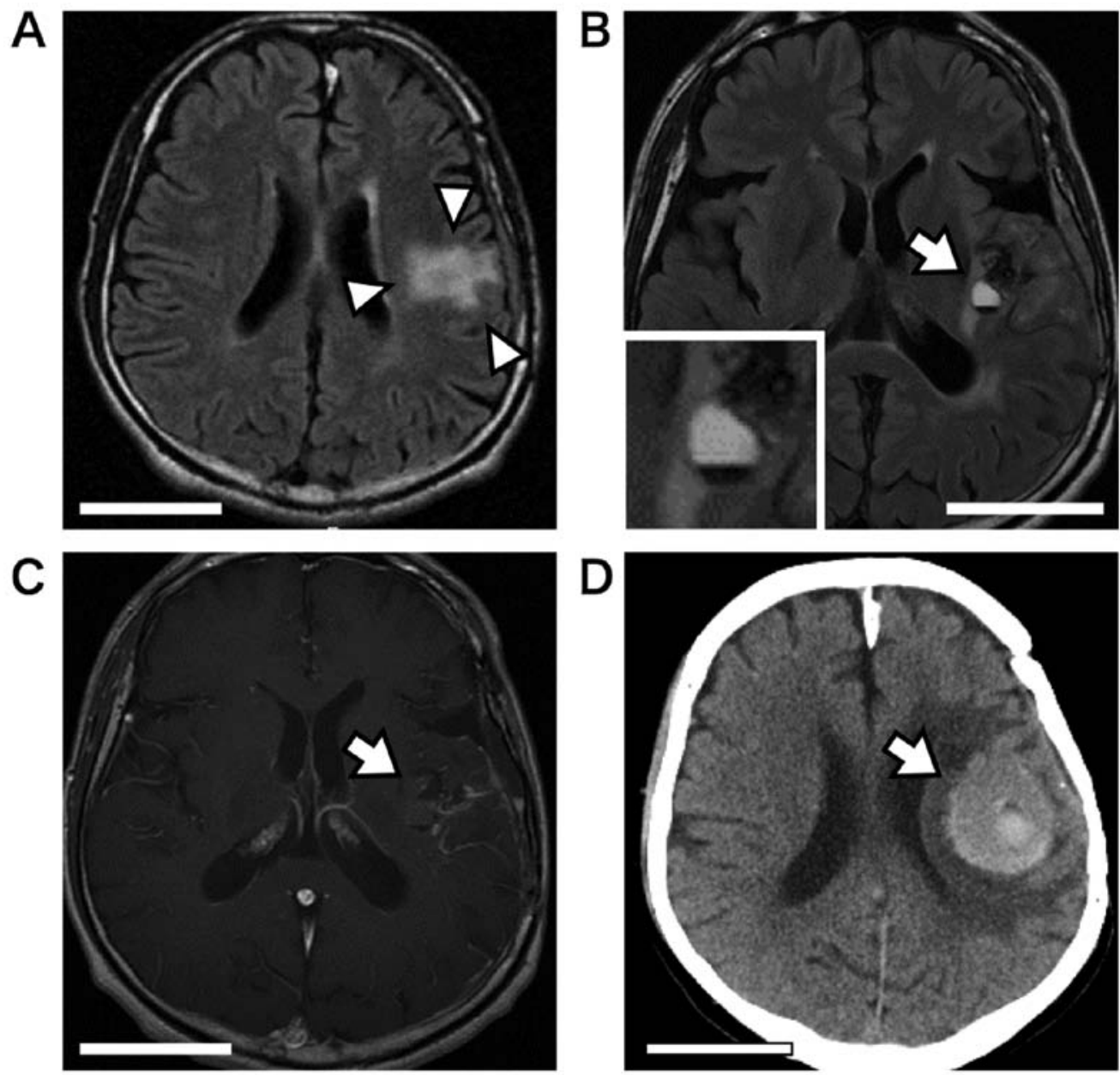

Figure 3. Case 3 (lesion 4). (A) At the age of 39, just after the initial treatment in the previous hospital, a high intensity lesion was observed on the FLAIR image as indicated by the arrow heads. (B and C) At the age of 50, a cystic lesion with a niveau on the FLAIR image (B) with slight contrast-enhancement (C) (arrow). The cystic component was highly intense in the top and lowly intense at the bottom (B inset). (D) The CT image of the first episode of acute hemorrhage as indicated by the arrow. Scale bar, $5 \mathrm{~cm}$.

vasculopathy, were observed in most of the cases (Fig. 4A). Tumor recurrence was not observed in all cases. In the totally resected lesions (lesions 1 and 3), the core of radiation necrosis consisted of RICM, which is a cavernous hemangioma-like component with thin-walled vessels (Fig. 4B and C: lesion 1, Fig. 4D and E: lesion 3). There were hemorrhages that permeated surrounding stroma of cavernous hemangioma-like lesions (Fig. 4F). A reactive increase in astrocyte number was observed in the perinecrotic or stromal hemorrhage area (Fig. 4G). Immunoreactivity for vascular endothelial growth factors (VEGF) was observed in the cytoplasm of the astrocytes in the perinecrotic hemorrhage area (Fig. $4 \mathrm{H}$ and I).

\section{Discussion}

Radiation necrosis is one of the major side effects of radiation therapy that usually occurs several months to years after radiation. The total incidence of radiation necrosis is estimated at $3-24 \%(1,15)$. The important risk factors of radiation necrosis are irradiation doses to brain tissue greater than $60 \mathrm{~Gy}$, a fraction size greater than 1.8-2.0 Gy, and subsequent administration of chemotherapy $(1,8,12,16)$. On the other hand, in most reported cases, RICM is observed at a frequency of about $6.7-41.2 \%$ in children with brain tumors long after radiation therapy (8-10).
RICMs were observed in the cases with fractionated radiation therapy with doses greater than 20 Gy or 30 Gy $(9,10,17)$. The higher the dosage, the shorter the latency (9). However, RICM in adult patients with glioma has been scarcely reported $(9,14)$.

The clinical characteristics of the patients are summarized in Table I. The histological diagnosis was oligoastrocytoma in 2 cases and anaplastic ependymoma in 1 case. The age at diagnosis ranged between 39 and 52 years (average, 47 years). The interval between the last irradiation and the occurrence of symptoms was 45-173 months (average, 107 months). Annual incidence of hemorrhage from RICM is reported to be about 4\%; approximately $40 \%$ of RICM patients are estimated to suffer from symptomatic hemorrhages $(14,18)$. The clinical courses and imaging findings of the presented cases of RICM with delayed radiation necrosis have the following characteristics. The ability of patients to perform daily activities deteriorated because of acute or chronic hemorrhages. Three lesions showed chronic enlargement of hematoma with uncontrollable perifocal edema. Although most radiation necrosis are responsive to steroid administration $(2,12,13)$, the perifocal edema of RICMs in the presented cases of radiation necrosis was steroid resistant and needed surgical interventions (case 1 and 3). Total resection of cavernous malformation, including necrotic tissues, appeared to be effective for improving neurological symptoms, such as seizure control. 
Table I. Summary of clinical characteristics of cases.

\begin{tabular}{|c|c|c|c|}
\hline Characteristics & Case 1 & Case 2 & Case 3 \\
\hline Lesion & Lesion 1 and 2 & Lesion 3 & Lesion 4 \\
\hline Age at diagnosis & 50 & 52 & 39 \\
\hline Sex & Female & Male & Male \\
\hline Histology & Oligoastrocytoma & Anaplastic ependymoma & Oligoastrocytoma \\
\hline Location & Left temporal and insular & Right occipital & Left frontal \\
\hline First irradiation & CyberKnife (35 Gy) & Conventional RT (60 Gy) & $\begin{array}{l}\text { Intraoperative RT + Conventional } \\
\text { RT ( } 56 \text { Gy) }\end{array}$ \\
\hline Additional irradiation & CyberKnife (30 Gy) & $\gamma$-Knife (20 Gy) & - \\
\hline $\begin{array}{l}\text { Last irradiation } \sim \text { symptom } \\
\text { onset (month) }\end{array}$ & 45 and 69 & 173 & 142 \\
\hline Clinical presentation & Chronic & Chronic & Acute \\
\hline Worsening of epilepsy control & Yes & Yes & No \\
\hline Outcome & MD & GR & $\mathrm{SD}$ \\
\hline
\end{tabular}

RT, radiation therapy; GR, good recovery; MD, moderate disabled; SD, severe disabled.
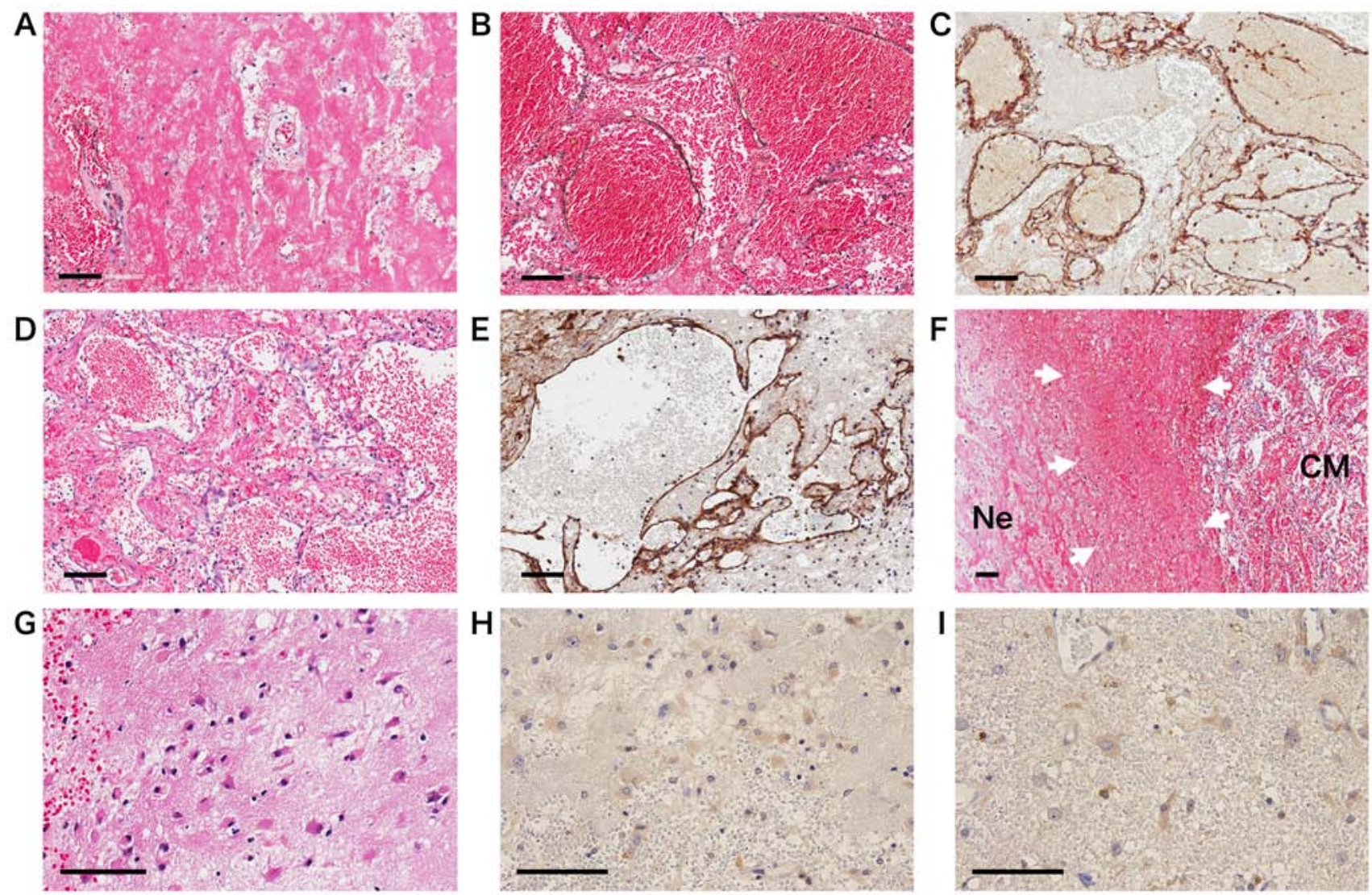

Figure 4. H\&E staining and immunohistochemistry of the surgical specimen. Bar=100 $\mu \mathrm{m}$. (A) Typical necrosis and hyalin exudation of the necrotic core of lesion 1 (H\&E, magnification, $\times 200$ ). (B-E) Cavernous malformation-like areas consist of thin and vulnerable vessels with telangiectasia and permeating hemorrhage of lesion 1 (H\&E, B and CD31, C) and lesion 3 (H\&E, D and CD31, E) (magnification, $\times 200)$. (F) Permeative hemorrhage areas (white arrows) are seen between the cavernous hemangioma-like lesion $(\mathrm{CM})$ and necrotic area $(\mathrm{Ne})$ of lesion 1 (H\&E magnification, $\times 100)$. (G) Peri-permeating hemorrhage areas in which large astrocytes are mixed with microhemorrhage (VEGF magnification, $\times 400)$. (H and I) Astrocytes are immunoreactive to VEGF. This immunoreactivity for VEGF is observed in peri-lesional interstitial hemorrhage areas of lesion 1 (magnification, $\times 400$ ).

It is important for appropriate treatments to distinguish tumor recurrence from RICM with radiation necrosis. The MRI study of delayed radiation necrosis with RICM showed chronic enlargement of heterogeneously enhanced lesions with perifocal edema and repeated hemorrhages with low intensity rim on $2^{*}(8)$. The presence of hypointense rim $\mathrm{T} 2 *$, which 
resembles the appearance of 'sporadic' (non-radiation-induced) cavernous malformations, could be helpful in differentiating RICM with delayed radiation necrosis from tumor recurrence. In fact, no tumor recurrence was found in any of the surgically-obtained specimens in this study.

Radiation-induced endothelial and glial damage may lead to microvasculopathy and telangiectasia, resulting in vascular insufficiency and infarction. This eventually culminates in gray and/or white matter necrosis (1-3). This hypoxia causes the upregulation of VEGF via the activation of hypoxia inducible factor- $1 \alpha$. The expression of VEGF produces leaky and fragile angiogenesis and subsequent perilesional edema in radiation necrosis (1). Immunoreactivity for VEGF is observed in the cytoplasm of astrocytes in the perinecrotic area. Upregulated production of VEGF may promote angiogenesis of vulnerable vessels, which then causes chronic or acute hemorrhages and strong perilesional edema.

Stereotactic radiotherapy could be an option for the treatment of unresectable gliomas, even as an initial treatment $(5-7,19)$. However, cases 1 and 2 received obvious overdose of radiation by an additional stereotactic radiotherapy and presented with repeated hemorrhages, medically uncontrollable symptomatic epilepsy, and cerebral edema. Although tumor did not recur, presented cases experienced disorders that affected their abilities to perform daily activities. Therefore, overdose radiation should be avoided in low grade glioma patients, who could potentially survive for a long period.

\section{Acknowledgements}

Not applicable.

\section{Funding}

No funding was received.

\section{Availability of data and materials}

All data generated or analyzed during this study are included in the published article.

\section{Author's contributions}

TO analyzed and interpreted data, and significantly contributed to the writing of the manuscript. TY, SK, TS, HN treated and operated on patients. SB performed the histological examination and diagnosis of the brain lesions. All authors were involved in the writing of the manuscript from the draft stage. All authors have read and approved the final revision.

\section{Ethics approval and consent to participate}

Each patient involved in this study provided written informed consent.

\section{Patient consent for publication}

Not applicable.

\section{Competing interests}

The authors declare that they have no competing interests.

\section{References}

1. Miyatake S, Nonoguchi N, Furuse M, Yoritsune E, Miyata T, Kawabata S and Kuroiwa T: Pathophysiology, diagnosis, and treatment of radiation necrosis in the brain. Neurol Med Chir (Tokyo) 55: 50-59, 2015.

2. Cheng KM, Chan CM, Fu YT, Ho LC, Cheung FC and Law CK: Acute hemorrhage in late radiation necrosis of the temporal lobe: Report of five cases and review of the literature. J Neurooncol 51: 143-150, 2001.

3. Nonoguchi N, Miyatake S, Fukumoto M, Furuse M, Hiramatsu R, Kawabata S, Kuroiwa T, Tsuji M, Fukumoto M and Ono K: The distribution of vascular endothelial growth factor-producing cells in clinical radiation necrosis of the brain: Pathological consideration of their potential roles. J Neurooncol 105: 423-431, 2011.

4. Motegi H, Kuroda S, Ishii N, Aoyama H, Terae S, Shirato H and Iwasaki Y: De novo formation of cavernoma after radiosurgery for adult cerebral arteriovenous malformation-case report. Neurol Med Chir (Tokyo) 48: 397-400, 2008.

5. Tanaka S, Shin M, Mukasa A, Hanakita S, Saito K, Koga T and Saito N: Stereotactic radiosurgery for intracranial gliomas. Neurosurg Clin N Am 24: 605-612, 2013.

6. Ogura K, Mizowaki T, Arakawa Y, Sakanaka K, Miyamoto S and Hiraoka M: Efficacy of salvage stereotactic radiotherapy for recurrent glioma: Impact of tumor morphology and method of target delineation on local control. Cancer Med 2: 942-949, 2013.

7. Ryken TC, Parney I, Buatti J, Kalkanis SN and Olson JJ: The role of radiotherapy in the management of patients with diffuse low grade glioma: A systematic review and evidence based clinical practice guideline. J Neurooncol 125: 551-583, 2015.

8. Kanda T, Wakabayashi Y, Zeng F, Ueno Y, Sofue K, Maeda T, Nogami $M$ and Murakami T: Imaging findings in radiation therapy complications of the central nervous system. Jpn J Radiol 36: 519-527, 2018.

9. Furuse M, Miyatake SI and Kuroiwa T: Cavernous malformation after radiation therapy for astrocytoma in adult patients: Report of 2 cases. Acta Neurochir (Wien) 147: 1097-1101, 2005.

10. Jain R, Robertson PL, Gandhi D, Gujar SK, Muraszko KM and Gebarski S: Radiation-induced cavernomas of the brain. AJNR Am J Neuroradiol 26: 1158-1162, 2005.

11. Lee JK, Chelvarajah R, King A and David KM: Rare presentations of delayed radiation injury: A lobar hematoma and a cystic space-occupying lesion appearing more than 15 years after cranial radiotherapy: Report of two cases. Neurosurgery 54: 1010-1013, 2004

12. Shah R, Vattoth S, Jacob R, Manzil FF, O'Malley JP, Borghei $P$, Patel BN and Cure JK: Radiation necrosis in the brain: Imaging features and differentiation from tumor recurrence. Radiographics 32: 1343-1359, 2012.

13. Rahmathulla G, Marko NF and Weil RJ: Cerebral radiation necrosis: A review of the pathobiology, diagnosis and management considerations. J Clin Neurosci 20: 485-502, 2013.

14. Cutsforth-Gregory JK, Lanzino G, Link MJ, Brown RD Jr and Flemming KD: Characterization of radiation-induced cavernous malformations and comparison with a nonradiation cavernous malformation cohort. J Neurosurg 122: 1214-1222, 2015.

15. Kawano H, Sato K, Hosotani K, Kubota T, Goya T, Arikawa S and Wakisaka S: Repeating hemorrhage with a long duration after radiotherapy for glioma: Radiological and histological observations. Brain Tumor Pathol 13: 85-92, 1996.

16. Yoshii Y: Pathological review of late cerebral radionecrosis. Brain Tumor Pathol 25: 51-58, 2008.

17. Winkler EA, Rutledge C, Ward M, Tihan T, Sneed PK, Barbaro N Garcia P, McDermott M and Chang EF: Radiation-induced cavernous malformation as a late sequelae of stereotactic radio surgery for epilepsy. Cureus 11: e2308, 2018.

18. Singla A, Brace O'Neill JE, Smith E and Scott RM: Cavernous malformations of the brain after treatment for acute lymphocytic leukemia: Presentation and long-term follow-up. J Neurosurg Pediatr 11: 127-132, 2013.

19. Hoffman LM, Plimpton SR, Foreman NK, Stence NV, Hankinson TC, Handler MH, Hemenway MS, Vibhakar R and Liu AK: Fractionated stereotactic radiosurgery for recurrent ependymoma in children. J Neurooncol 116: 107-111, 2014. 